\title{
Niveles de mercurio en sedimentos de la zona costera de El Oro, Ecuador
}

\section{Mercury in sediments at El Oro province, Ecuador}

\author{
Alfonso Marín ${ }^{1}$, Victor Hugo Gonzalez ${ }^{1}$, Byron Lapo ${ }^{1}$, Estefania Molina ${ }^{1}$ \& Mairin Lemus ${ }^{1,2,3}$, \\ ${ }^{1}$ Universidad Técnica de Machala, Machala, Ecuador \\ 2 Programa Prometeo Senescyt \\ 3Universidad de Oriente, Cumaná, Venezuela \\ Corresponding author: mlemus88@gmail.com
}

\begin{abstract}
RESUMEN
Se analizó el contenido total de $\mathrm{Hg}$ en sedimentos superficiales en tres localidades de la zona costera de la Provincia de El Oro, Ecuador. En cada localidad se seleccionaron 9 zonas de muestreo, en los cuales se evaluó: sólidos totales (ST), humedad $(\mathrm{H})$, materia orgánica $(\mathrm{MO})$ y $\mathrm{Hg}$ total. La concentración del $\mathrm{Hg}$ fue determinada por espectrofotometría de absorción atómica, por generación de hidruros con atomización en una celda de cuarzo. Las concentraciones de Hg presentaron diferencias significativas entre las localidades, con la mayor concentración en el estero Huaylá (intervalo: 6,57-7,61 mg kg $\mathrm{mg}^{-1}$ y la menor en Bajo Alto (intervalo: 3,46-4,83 $\mathrm{mg} \mathrm{kg}^{-1}$ ). Los niveles de $\mathrm{Hg}$ en los sedimentos sugieren una evidente incorporación antrópica en las tres localidades del perfil costero de la provincia de El Oro, lo cual representa un riesgo para la biota y la salud humana.
\end{abstract}

Palabras claves: metal pesado, contaminación, zona costera, materia orgánica.

\begin{abstract}
The total content of $\mathrm{Hg}$ was evaluated in surface sediments at three localities in the coastal area of the province of El Oro, Ecuador. In each locality nine area of sampling were established, in which the total solids (ST), humidity (H), organic matter (MO) and $\mathrm{Hg}$ was analyzed. The $\mathrm{Hg}$ concentration was determined by atomic absorption spectrophotometry, by hydride generation with atomization in a quartz cell. The concentrations of $\mathrm{Hg}$ showed significant differences between localities, with the largest concentration in the Huayla estuary (range: $6,57-7,61 \mathrm{mg} \mathrm{kg}^{-1}$ ) and the lowest in Bajo Alto (range: $3,46-4,83 \mathrm{mg} \mathrm{kg}^{-1}$ ). The levels of $\mathrm{Hg}$ in sediments suggest a clear anthropic incorporation in the three localities of the coastal profile of the province of El Oro, which represent a risk to the biota and human health.
\end{abstract}

KEYwORDS: Heavy metal, contamination, sediment, coast, organic matter.

\section{INTRODUCCIÓN}

Dentro de los ecosistemas acuáticos, los sedimentos funcionan como trampas naturales de xenobióticos y constituyen el principal reservorio de la mayoría de las sustancias de origen antrópico. Particularmente los metales, presentan una elevada densidad (mayores de $7 \mathrm{~g} \mathrm{~cm}^{-1} \mathrm{a}$ excepción del aluminio) y pasan a formar parte de los sedimentos. El mercurio $(\mathrm{Hg})$ es uno de los elementos más tóxicos y la formación de especies solubles en agua son limitadas. El Hg suele quedar atrapado en los sedimentos, permaneciendo en formas que son poco biodisponibles y difíciles de remover, debido a la presencia de materia orgánica y sulfuros que le proporcionan una condición reductora (Vane et al. 2009). La forma particulada constituye más del $90 \%$ del mercurio total en sistemas de agua dulce, estuarios o zonas costeras (Schäfer et al. 2006). No obstante, un pequeño porcentaje del $\mathrm{Hg}$ enlazado a las partículas de sedimento de forma inestable, puede cambiar su forma iónica y estar biodisponible para biota, haciendo posible su flujo hacia la columna de agua y a la cadena alimentaria (Monikh et al. 2013, Thera \& Rumbold 2014). Los principales problemas ambientales en los cuerpos de agua se asocian con la descarga de efluentes urbanos y de las actividades industriales que han tenido como consecuencia un deterioro en la salud de los ecosistemas, poniendo en 
peligro la abundancia y biodiversidad de especies y la calidad de vida de los seres humanos, un ejemplo de ello fue la tragedia de Minamata ocurrida en 1953 (Eto 2000).

Dado estos antecedentes, los análisis químicos de los sedimentos adquieren un gran valor científico en el estudio de los ecosistemas, pues con ellos se logra obtener un registro histórico del tipo de contaminación que ha tenido lugar en las zonas próximas a su recorrido, y al interaccionar con las corrientes de agua ponen en circulación los contaminantes retenidos, alterando la dinámica general de la masa de agua (Mariani \& Pompêo 2008).

El monitoreo de los metales pesados es una herramienta utilizada en muchos países desarrollados para evaluar la calidad de los ecosistemas, pero no en todos los países donde se sospecha que existe la presencia de metales pesados se evalúan. La provincia de El Oro, tiene actividades de tipo acuícola, agrícola y minero; actividades que han venido deteriorando los ecosistemas, con un creciente enriquecimiento de sustancias orgánicas e inorgánicas de los sedimentos. Son pocos y muy puntuales los estudios que han evaluado su efecto sobre los ecosistemas en la provincia de El Oro. Tarras-Wahlberg et al. (2001), describen el efecto de la minería a pequeña escala en el sureste del Ecuador, particularmente la minería en Portovelo-Zaruma y su impacto sobre el río Puyango. Para el año 1999, se estimó que el componente sólido de los relaves que escaparon hacia los ríos ascendió a 160000 toneladas, 33000 cargadas de mercurio y otros metales y el resto de material estaba constituido por lodos de cianuros y metales provenientes de plantas de cianuración. Las concentraciones determinadas en el sedimento en áreas de la minería mostraron valores de $\mathrm{Hg}$ en un rango de 0,08 y $0,99 \mathrm{mg} \mathrm{Kg}^{-1}$ para el año $1998 \mathrm{y}$ 1999, mientras que para el sedimento en suspensión de $\mathrm{Hg}$ (fracción $<0,45 \mu \mathrm{m}$ ) estuvo comprendida entre 0,8 y 2,3 $\mathrm{mg} \mathrm{Kg}{ }^{-1}$. Otro, estudio realizado para la misma fecha han evaluado los impactos ambientales de la minería en el medio ambiente y el hombre (Harari et al. 2012). Betancourt et al. (2005) determinaron que en las plantas procesadoras del oro, los niveles de $\mathrm{Hg}$ en agua alcanzan valores de $250 \mathrm{ng}$ de $\mathrm{Hg}$ $\mathrm{L}^{-1}$, que aun cuando no supera los límites permisibles para agua de consumo $\left(1 \mu \mathrm{g}\right.$ de $\left.\mathrm{Hg} \mathrm{L}^{-1}\right)$, el $10 \%$ de la población supera los límites en sangre de $4 \mu \mathrm{g}$ de $\mathrm{Hg} \mathrm{L}^{-1}$ (WHO, 1991).

No existen registros científicos de monitoreos de agroquímicos y metales pesados en la zona costera de la provincia de El Oro, a pesar de que hay conocimiento de que los agroquímicos usados en las bananeras son descargados directamente hacia la zona costera a través de los ríos y por arrastre de las lluvias. Los agroquímicos peligrosos que contienen $\mathrm{Hg}$ fueron prohibidos en Ecuador, cuando el país se adhiere al Convenio de Rotterdam 2005, sin embargo, dada la persistencia del $\mathrm{Hg}$ presente en los pesticidas, estos pueden estar presentes y su registro es fundamental para determinar la salud de los ecosistemas. Actualmente, se han determinado severos efectos sobre la calidad de los ecosistemas costeros. Los principales impactos de las descargas de la minería en la provincia de El Oro se han relacionado con una severa pérdida de la biodiversidad, particularmente en los ríos Puyango, Siete, Gala y Río Chico y una significativa bioacumulación de metales pesados en la biota. También, como consecuencia de estas actividades hay un severo efecto en la calidad del agua, tanto para el consumo humano como para el riego (Sandoval 2001).

Dado que es evidente la gran cantidad de desechos domésticos, agrícolas y mineros que son descargados a los cuerpos de agua en la provincia de El Oro sin ningún tipo de tratamiento, en éste estudio se realiza una evaluación de los niveles de $\mathrm{Hg}$ en tres localidades de la zona costera de la provincia de El Oro.

\section{MATERIALES Y METODOS}

Área de ESTUdio

La investigación se realizó en tres localidades del perfil costanero de la provincia de El Oro: Bajo alto, El Coco y el estero Huaylá (Fig. 1, Tabla 2). En cada una de las localidades se recolectaron sedimentos en tres zonas con tres réplicas cada una. La localidad de Bajo Alto pertenece a la parroquia Tendales del cantón El Guabo y es una playa de uso recreativo. El Coco pertenece al cantón Machala a 4,5 millas náuticas del Archipiélago de Jambelí, y también se usa con fines recreativos. El estero Huaylá está ubicado al suroeste del cantón Machala, caracterizado por la circulación embarcaciones de pesca artesanal, de turismo, de carga y grandes gabarras que desembarcan en el muelle ubicado en las cercanías del estero.

\section{RECOLECCIÓN Y PRE-TRATAMIENTO DE LAS MUESTRAS}

Las muestras de sedimento recolectadas, se almacenaron en bolsas plásticas con cierre hermético previamente rotuladas, se trasladaron al laboratorio donde se secaron a temperatura ambiente durante 96 horas. Para garantizar el secado total, se utilizó una estufa a una temperatura de $60{ }^{\circ} \mathrm{C}$ durante $72 \mathrm{~h}$. Una vez secas se molieron en un mortero y luego se tamizaron en un tamiz de $65 \mu \mathrm{m}$.

\section{DETERMINACIÓN DE MATERIA ORGÁNICA}

El contenido de materia orgánica, se determinó por el método descrito por De la Lanza (1980); el cual se fundamenta en la mineralización total de la materia orgánica que se encuentra contenida en los sedimentos marinos. Se calcinó un gramo de sedimento en una mufla a $450^{\circ} \mathrm{C}$, de tal manera que la fracción libre de materia orgánica se cuantifica por la pérdida de peso debido a la oxidación de materia orgánica en la muestra a $\mathrm{CO}_{2}$. 


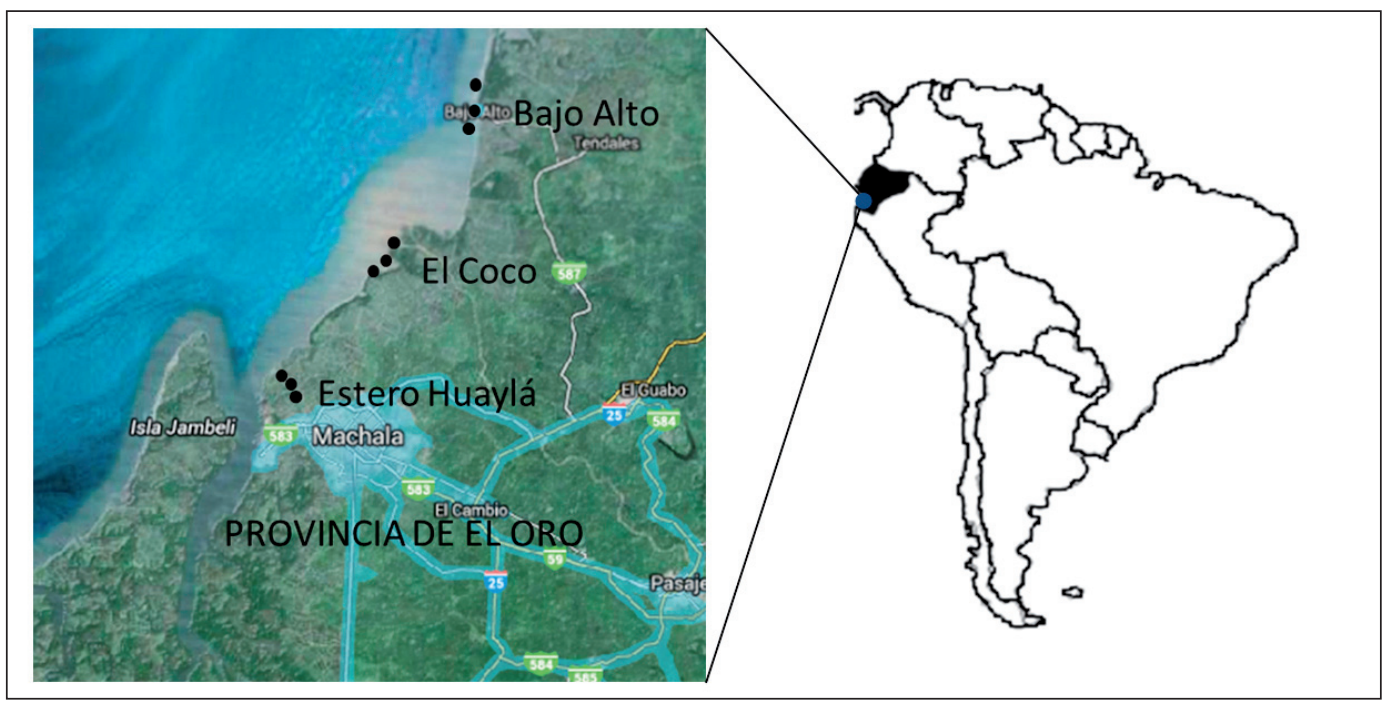

FIGURA 1. Área de estudio mostrando la ubicación de los sitios de recolección de los sedimentos en la zona costera de la provincia de el oro, ecuador.

FIGURE 1. Sampling sites of collection sites of sediments at marine coast of the El Oro, Ecuador.

TABLA 1. Análisis de Hg en sedimento certificado MESS-3

TABLE 1. Analysis of $\mathrm{Hg}$ in sediment MESS-3 certificate

\begin{tabular}{lc}
\hline \multicolumn{1}{c}{ Metal } & $\mathrm{Hg}$ \\
\hline Replica1 & 0,10 \\
Replica 2 & 0,10 \\
Replica 3 & 0,11 \\
\hline Promedio & $0,10 \pm 0,064$ \\
Valor recomendado & $0,091 \pm 0,009$ \\
\hline
\end{tabular}

\section{DETERMINACIÓN DE HUMEDAD Y SÓLIDOS TOTALES}

Las muestras de sedimento se depositaron en capsulas de porcelana, previamente lavadas con $\mathrm{H}_{2} \mathrm{NO}_{3}$ al $10 \%$ y agua desionizada $15 \mathrm{~m} \Omega$, y se instalaron en una estufa a $65^{\circ} \mathrm{C}$ por un tiempo de 72 horas o hasta lograr un peso constante. Posteriormente, se mantuvieron en un desecador durante 24 hrs., siguiendo la metodología ISO 11465 (1993).

\section{DETERMINACIÓN DE MERCURIO}

A 0,5gr. de sedimento se le agregaron $10 \mathrm{ml}$ de $\mathrm{HNO}_{3}$ concentrado de alta pureza; se les colocó un pequeño embudo en la parte superior del Erlenmeyer para impedir la pérdida de muestra y se dejó en digestión durante una noche a temperatura ambiente. Después, se procedió a digerir a una temperatura de $60^{\circ} \mathrm{C}$ durante 1 hora; luego, la temperatura se aumentó progresivamente hasta $100^{\circ} \mathrm{C}$, manteniéndola durante tres horas. Posteriormente, se le agregaron $2,5 \mathrm{ml}$ de $\mathrm{H}_{2} \mathrm{SO}_{4}$ concentrado y $1 \mathrm{ml}$ de $\mathrm{HCl}$ concentrado (ambos de alta pureza) y se dejó digerir por 3 horas más (Márquez et al. 2012). Seguidamente, se centrifugaron a $3000 \mathrm{~g}$ por 45 min. Las muestras se enrasaron a $25 \mathrm{ml}$ y se guardaron en refrigeración hasta que se analizaron. Para la determinación de $\mathrm{Hg}$ se tomaron $10 \mathrm{ml}$ de la muestra previamente digerida, se le agregó algunas gotas de $\mathrm{KMnO}_{4}$ al $5 \% \mathrm{~m} / \mathrm{V}$ libre de mercurio, hasta que se observó una coloración rosada permanente. $\mathrm{El}$ exceso de $\mathrm{KMnO}_{4}$ se tituló con clorhidrato de hidroxilamina al 5\% m/V (Rojas et al. 2002). El mercurio se determinó utilizando generación de hidruros con atomización en una celda de cuarzo, en un Espectrometro de Absorción Atómica Vapor-Frío en un equipo Shimadzu, AA 7000.

Para determinar la veracidad y la precisión del método, se realizó la trazabilidad de los resultados con un material de referencia para metales (MESS-3 Marine Sediment Reference Materials for Trace Metals and other Constituents), los cuales muestran los resultados en la tabla 1 .

\section{AnÁLisis Estadístico}

Los análisis estadísticos para los niveles de Hg y parámetros físico-químicos obtenidos, se realizaron utilizando el programa de STATGRAPHICS Centurión XVI. Se realizaron comparaciones de la desviación estándar y medianas, además de las pruebas de Kolmogorov-Smimov, Kruskal-Wallis y la Mediana de Mood. 


\section{RESULTADOS Y DISCUSIÓN}

En el presente estudio se evaluaron los niveles de $\mathrm{Hg}$ en sedimentos superficiales de la zona costera de la provincia de El Oro, en una extensión de costa de $203 \mathrm{Km}$ aproximadamente. Los sedimentos se caracterizaron por presentar elevados niveles de materia orgánica $(13,99 \%$ al $24,60 \%$ ) y con un porcentaje de sedimento de la fracción menor que $65 \mu \mathrm{m}$ de 11,13 y $14,84 \%$. El contenido de sólidos totales osciló entre un $64,36 \%-80,53 \%$, y las mayores concentraciones se encontraron en Estero Huaylá (Tabla 2). Estos datos sugieren que hay una alta carga de materia orgánica en la zona costera, atributo característico de los ecosistemas de manglares. Las condiciones anaerobias dominantes en este tipo de humedales, generan suelos con mucha turba (Macfarlene et al. 2007). Toda la zona costera de la provincia de El Oro está dominada por ecosistemas de manglares, en los cuales el efecto antrópico está determinado por las descargas residuales de la ciudad de Machala, particularmente la que ocurren en el Estero Huaylá.

Se determinó que existen diferencias significativas en los niveles de $\mathrm{Hg}$ en los sedimentos de los tres sitios de muestreo de cada una de las localidades $\left(\mathrm{KW}_{\mathrm{BA}}=8,11 ; \mathrm{P}=\right.$ 0,$\left.000 ; \mathrm{KW}_{\mathrm{CC}}=10,88 ; \mathrm{P}=0,000 ; \mathrm{KW}_{\mathrm{EH}}=23,00 ; \mathrm{P}=0,000\right)$. En BA la mayor concentración fue de 4,83 $\pm 0,65 \mathrm{mg}$ de $\mathrm{Hg} \cdot \mathrm{Kg}^{-1}$, en el Coco de 4,40 mg de Hg. $\mathrm{Kg}^{-1}$ y en Huaylá de 7,6182 mg de Hg.Kg-1 (Fig. 2). Las diferencias en los niveles de mercurio de los sedimentos superficiales en una misma localidad demuestran una distribución del mercurio asociada a las características particulares de parches, relacionada con sedimentos más finos y mayor presencia de materia orgánica, esto supone microambientes apropiados para una mayor adsorción del Hg.

Comparando los resultados de $\mathrm{Hg}$ en sedimento superficial entre localidades se observa que los sedimentos de BA y el $\mathrm{CC}$ fueron significativamente menores $(\mathrm{KW}=17,90$, $\mathrm{P}=0,000$ ) con valores de 3,97 $\pm 0,73 \mathrm{mg}$ de $\mathrm{Hg}$. $\mathrm{Kg}^{-1}$ y 4,09 $\pm 0,49 \mathrm{mg}$ de $\mathrm{Hg} . \mathrm{Kg}^{-1}$ respectivamente, mientras que el Estero Huaylá mostró la máxima concentración con un valor de 7,08 $\pm 1,15 \mathrm{mg}$ de $\mathrm{Hg}$. $\mathrm{Kg}^{-1}$. Estos resultados demuestran que evidentemente el estero presenta aproximadamente $40 \%$ más mercurio que los ecosistemas anteriores.

Estos niveles de mercurio determinan un gran efecto antrópico en los ecosistemas, no obstante, el Estero Huaylá es el más perturbado. Los mayores niveles de $\mathrm{Hg}$ en este estero podrían ser explicados por las corrientes marinas y por la deposición del $\mathrm{Hg}$ en la columna de agua, que ocurre durante la pleamar y baja mar, arrastrado sedimentos enriquecidos desde la costa hasta el interior del estero, que posteriormente es atrapado por la materia orgánica que se encuentra en mayor proporción (Tabla 1) en relación a las otras localidades. No se descarta que las descargas de los agroquímicos residuales con altos niveles de $\mathrm{Hg}$ del Cantón Machala que se habían realizado años atrás en las plantaciones de banano hayan contribuido a la presencia de este metal en los ecosistemas; aunque su aplicación fue suspendida por el Acuerdo Ministerial No 0112, publicado en el Registro Oficial No 64 con fecha 12 de Noviembre de 1992 (Rasabala 2008). Estos aspectos podrían explicar los mayores niveles de $\mathrm{Hg}$ en el estero, porque aunque las otras localidades están más expuestas a las corrientes marinas y son las que reciben las descargas de los ríos que provienen de las cuencas altas donde se realiza la minería, presentan menores concentraciones de Hg que el Estero Huaylá.

La distribución de $\mathrm{Hg}$ en los sedimentos de las localidades estudiadas, puede ser explicada en gran medida por la presencia de la materia orgánica, pues se determinó una relación positiva y significativa entre los niveles de $\mathrm{Hg}$ $(\mathrm{r}=0,82 ; \mathrm{Fs}=55,30 ; \mathrm{p}=0,000)$ y el contenido de materia orgánica en los sedimentos (Fig. 3).

La fuerte unión del $\mathrm{Hg}$ a la MO se atribuye a la coordinación del mercurio a los grupos sulfidrilos de la materia orgánica, que están presentes en concentraciones mucho más altas que las concentraciones de mercurio y se encuentran en la mayoría de las aguas. También el Hg puede precipitar en forma de sulfuro mercúrico, un sólido altamente insoluble. Este fuerte enlazamiento del metal a la $\mathrm{MO}$ es dado por una elevada constante de estabilidad entre el azufre de la materia orgánica y el mercurio (RS-Hg) (Skyllberg et al. 2000, Ravichandran 2004). La MO parece desempeñar un papel clave en la reducción fotoquímica de mercurio iónico

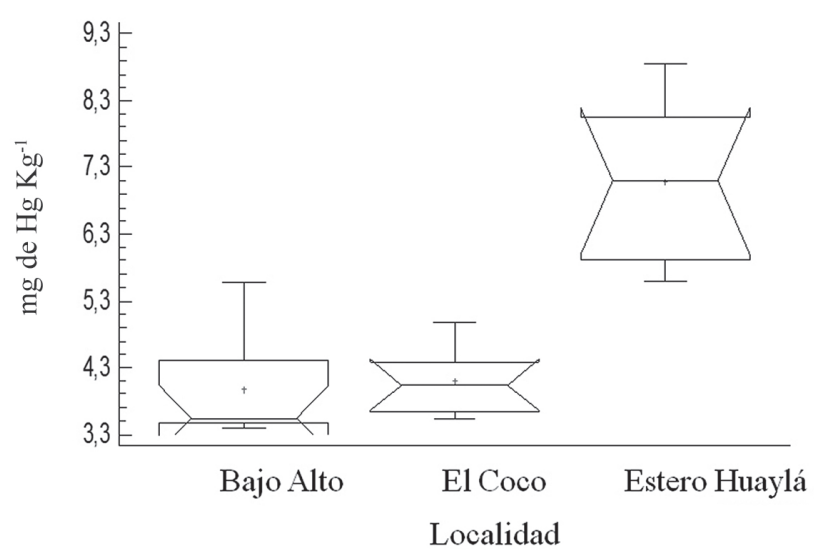

Figura 2. Concentración de $\mathrm{Hg}$ (mg. $\mathrm{kg}^{-1}$ en masa seca) en sedimentos superficiales de tres localidades del perfil costanero de la provincia de El Oro.

FIGURE 2. Mercury concentration (mg. $\mathrm{kg}^{-1}$ dry weight) in surface sediments of three localities at marine coast El Oro, Ecuador. 


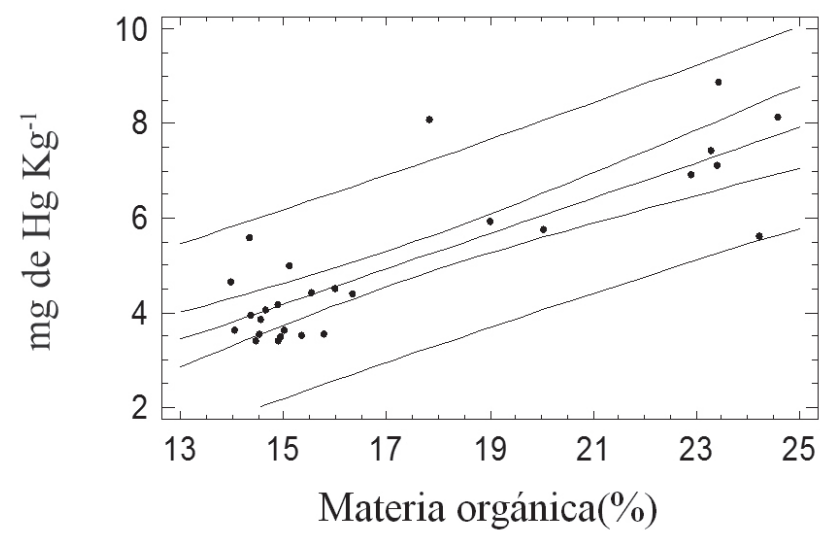

Figura 3. Regresión lineal entre $\mathrm{Hg}$ y la materia orgánica de sedimento de la zona costera de la provincia de El Oro.

FIGURE 3. Relationship between $\mathrm{Hg}$ and organic matter of sediment in the coastal area of the province of El Oro. a mercurio elemental y la posterior reoxidación del mercurio elemental a mercurio iónico. Este proceso determina la volatilización y la biodisponibilidad de mercurio para los organismos, por lo que la MO determina la producción y la bioacumulación de metilmercurio.

Los niveles de $\mathrm{Hg}$ en la zona costera de la provincia de El Oro, se encuentran en el mismo orden de magnitud a las realizadas en zonas profundas en el litoral de la zona estuarina externa del Golfo de Guayaquil (06-1 mg Kg ${ }^{-1}$ ) y el canal de Jambelí (0,94 - 1,04 mg Kg-1) (Chalén 2010). No obstante, valores en un rango de 3,67-7,61 $\mathrm{mg} \mathrm{Kg}^{-1}$ están asociados a zonas mucho más perturbadas como es el caso de las zonas costeras, que representa el sitio de entrada de los metales a través de las descargas fluviales y los ríos y son más rápidamente atrapadas por los sedimentos y la materia orgánica.

TABla 2. Concentración promedio de $\mathrm{Hg}$ y $\mathrm{Pb}\left(\mathrm{mg} \mathrm{kg}^{-1}\right.$ en peso seco) y contenido promedio de solitos totales, humedad y materia orgánica en la zona costera de la Provincia de El Oro, Ecuador.

TABLE 2. Mercury and cadmium concentration (mg.kg-1 dry weight) total solids, moisture and organic matter at marine coast El Oro, Ecuador.

\begin{tabular}{|c|c|c|c|c|c|c|c|c|}
\hline ZONA & LOCALIDAD & UBICACIÓN & & $\mathrm{Hg}$ & $\begin{array}{c}\text { Sólidos } \\
\text { Totales } \\
(\%)\end{array}$ & $\begin{array}{l}\text { HUMEDAD } \\
(\%)\end{array}$ & $\begin{array}{c}\text { Materia } \\
\text { OrgÁNICA } \\
(\%)\end{array}$ & $\begin{array}{c}\text { CARACTERÍSTICAS DE } \\
\text { LA ZONA }\end{array}$ \\
\hline \multirow{3}{*}{ BAJO ALTO } & 1 & $\begin{array}{c}3^{\circ} 5^{\prime} 6.94 \prime \\
79^{\circ} 53 ' 45.11^{\prime \prime}\end{array}$ & & $\begin{array}{l}4,83 \\
0,65\end{array}$ & $65,360,33$ & $\begin{array}{r}34,64 \\
0,33\end{array}$ & $\begin{array}{r}14,35 \\
0,85\end{array}$ & Zona de Manglar \\
\hline & 2 & $\begin{array}{c}3^{\circ} 5{ }^{\prime} 33.73 " \\
79^{\circ} 53 ' 51.84 "\end{array}$ & $\begin{array}{l}\bar{X} \\
\text { SD }\end{array}$ & $\begin{array}{l}3,62 \\
0,27\end{array}$ & $66,21 \quad 0,40$ & $\begin{array}{r}33,79 \\
0,40\end{array}$ & $\begin{array}{r}14,36 \\
0,79\end{array}$ & Zona de Manglar \\
\hline & 3 & $\begin{array}{c}3^{\circ} 6^{\prime} 1.38^{\prime \prime} \\
79^{\circ} 54^{\prime} 1.28^{\prime \prime}\end{array}$ & $\begin{array}{l}\bar{X} \\
\mathrm{SD}\end{array}$ & $\begin{array}{l}3,46 \\
0,05\end{array}$ & $65,01 \quad 0,55$ & $\begin{array}{r}34,99 \\
0,55\end{array}$ & $\begin{array}{r}15,36 \\
0,25\end{array}$ & Zona de costera \\
\hline \multirow{3}{*}{ EL COCO } & 1 & $\begin{array}{l}3^{\circ} 13^{\prime} 28.74^{\prime \prime} \\
79^{\circ} 59^{\prime} 35.49^{\prime \prime}\end{array}$ & $\begin{array}{l}\bar{X} \\
\text { SD }\end{array}$ & $\begin{array}{l}4,40 \\
0,56\end{array}$ & $64,36 \quad 0,47$ & $\begin{array}{r}35,64 \\
0,47\end{array}$ & $\begin{array}{r}14,35 \\
0,92\end{array}$ & Zona de Costa \\
\hline & 2 & $\begin{array}{c}3^{\circ} 13^{\prime} 36.60^{\prime \prime} \\
79^{\circ} 59^{\prime} 44.50^{\prime \prime}\end{array}$ & $\begin{array}{l}\bar{X} \\
\text { SD }\end{array}$ & $\begin{array}{l}4,09 \\
0,52\end{array}$ & $64,730,16$ & $\begin{array}{r}35,27 \\
0,16\end{array}$ & $\begin{array}{r}13,99 \\
0,36\end{array}$ & Zona de Costa \\
\hline & 3 & $\begin{array}{c}3^{\circ} 13^{\prime} 45.96^{\prime \prime} \\
79^{\circ} 59^{\prime} 49.43^{\prime \prime}\end{array}$ & $\begin{array}{l}\bar{X} \\
\mathrm{SD}\end{array}$ & $\begin{array}{l}3,77 \\
0,33\end{array}$ & $64,73 \quad 0,78$ & $\begin{array}{r}35,27 \\
0,78 \\
\end{array}$ & $\begin{array}{r}14,89 \\
0,24 \\
\end{array}$ & Zona de Camaronera \\
\hline \multirow{3}{*}{ HUAYLÁ } & 1 & $\begin{array}{l}3^{\circ} 16^{\prime} 23.60^{\prime \prime} \\
80^{\circ} 00^{\prime} 2.75^{\prime \prime}\end{array}$ & $\begin{array}{l}\bar{X} \\
S D\end{array}$ & $\begin{array}{l}7,61 \\
1,08\end{array}$ & $75,630,63$ & $\begin{array}{r}24,37 \\
0,63\end{array}$ & $\begin{array}{r}23,44 \\
0,29\end{array}$ & Estero \\
\hline & 2 & $\begin{array}{l}3^{\circ} 16^{\prime} 23.88^{\prime \prime} \\
80^{\circ} 0 ’ 6.77^{\prime}\end{array}$ & $\begin{array}{l}\bar{X} \\
\text { SD }\end{array}$ & $\begin{array}{l}7,05 \\
1,30\end{array}$ & $75,34 \quad 1,16$ & $\begin{array}{c}24,66 \\
1,6\end{array}$ & $\begin{array}{r}24,6 \\
0,67\end{array}$ & Estero \\
\hline & 3 & $\begin{array}{l}3^{\circ} 16^{\prime} 24.31^{\prime \prime} \\
80^{\circ} 00^{\prime} 10.52^{\prime \prime}\end{array}$ & $\begin{array}{l}\bar{X} \\
\text { SD }\end{array}$ & $\begin{array}{l}6,57 \\
1,28\end{array}$ & $80,53 \quad 1,51$ & $\begin{array}{r}19,47 \\
1,51\end{array}$ & $\begin{array}{r}17,83 \\
1,11\end{array}$ & Estero \\
\hline
\end{tabular}


Gayana 80(2), 2016

TABla 3. Concentraciones de Hg total en sedimentos de distintos ecosistemas acuáticos. Las concentraciones se refieren a peso seco.

TABLE 3. Concentrations absolute $\mathrm{Hg}$ in different aquatic ecosystem sediments concentrations refers the dry weight.

\begin{tabular}{lcc}
\hline Sitio & $\begin{array}{c}\text { Hg Total EN SEdimentos } \\
\left(\mathrm{mg} . \mathrm{Kg}^{-1}\right)\end{array}$ & Referencia \\
\hline Puerto Montevideo, Uruguay & $0,3-1,3$ & Muniz et al. 2004 \\
Río Negro (planta de cloro álcali) & $0,8-3,4$ & Arribére et al. 2003 \\
Canal Oeste, Ensenada & 6,8 & Peluso et al. 2011 \\
Riachuelo, Buenos Aires & 1.9 & Peluso et al. 2011 \\
Río Negro (Amazonas), Brasil & $70-270$ & Bisinoti et al. 2007 \\
Mina Del Diablo Rojo, Suroeste De Alaska- EEUU & 78,40 & Bloom et al. 2003 \\
Rio Puyango, Ecuador (material es suspensión) & $0,8-4-8$ & Tarras-Wahlberg et al. 2001 \\
Rio Puyango, Ecuador (sedimentos) & $0,22-0,85$ & Tarras-Wahlberg et al. 2001 \\
Golfo de Guayaquil y Canal de Jambelí, Ecuador & $0,6-1,0$ & Chalén 2010 \\
Bajo Alto, El Oro- Ecuador & 3,97 & Este estudio \\
El Coco- El Oro- Ecuador & 4.09 & Este estudio \\
Estero Huaylá, El Oro Ecuador & 7,08 & Este estudio \\
\hline
\end{tabular}

Adicionalmente las concentraciones de $\mathrm{Hg}$ en la zona costera son comparables a otros sitios (Tabla 3), donde se ha determinado intervención antrópica asociada a efluentes con cargas significativas del metal, como por ejemplo el estudio realizado cerca de una planta de cloro álcali donde las concentraciones están entre un rango de 0,8-3,4 mg $\mathrm{Kg}^{-1}$ (Arriberé et al. 2003) también otro estudio donde existe un gran impacto de efluentes con diferentes orígenes, muestran niveles de $6,8 \mathrm{mg}$ de $\mathrm{Hg} \mathrm{Kg}^{-1}$ (Peluso et al. 2011). No obstante, el $\mathrm{Hg}$ en sedimento en la zona costera de la Provincia de El Oro se encuentra por debajo de los niveles mostrados en áreas impactadas directamente por la minería como es el caso del río Negro en el Amazonas, Brazil que muestra concentraciones entre 70-270 mg de $\mathrm{Hg} \mathrm{Kg}^{-1}$ (Bisinoti et al. 2007).

Los valores de $\mathrm{Hg}$ obtenidos en la presente investigación superan los señalados por normativas internacionales de calidad de sedimentos, la canadiense señala $0,13 \mathrm{mg} \mathrm{Hg}$. $\mathrm{Kg}^{-1}$ (ENVIRONMENT CANADA 1990), mientras que la norma Holandesa propone $0,3 \mathrm{mg} \mathrm{Hg} \cdot \mathrm{Kg}^{-1}$ (IADC/CEDA 1992). También es importante considerar la concentración de efecto probable (PEL), propuesta por los EEUU, la cual señala que dosis superiores a $0,70 \mathrm{mg} \cdot \mathrm{Kg}^{-1}$, pueden tener probables efectos sobre la biota; en este sentido se puede asumir que los sedimentos de la zona costera de la provincia de El Oro podrían representar un riesgo para la salud de los ecosistemas, incluyendo al hombre.

En el caso de la salud humana en Ecuador se ha demostrado que los niveles de mercurio en sangre de mujeres de una localidad minera, muestran valores de $2,7 \mu \mathrm{g} \mathrm{L}^{-1}$ con un rango entre 0,26 y 9,8, valores que son comparables con mujeres de zonas mineras de china. Ambos niveles son muy superiores a mujeres provenientes de otros países como la república Checa, Croacia, Eslovaquia, Eslovenia y otros (Pawlas et al. 2013). Por tanto, existe una bioacumulación y biomagnificación significativa de mercurio presente en los ecosistemas de Ecuador que está afectando a la población y que requiere ser evaluada con un mayor nivel de detalle.

\section{AGRADECIMIENTOS}

Los autores agradecen al Proyecto Prometeo de la Secretaria de Educación Superior, Ciencia Tecnología e Innovación de la República del Ecuador y a la Universidad Técnica de Machalaasí como también el Consejo de Investigación de la Universidad de Oriente

\section{BIBLIOGRAFÍA}

Arribere, M., Guevara, S., Sanchez, R., Gil, M., Ross, G., Daurade, L., Fajon, V., Horvat, M., Alcalde R. \& KestelMAN, A. 2003. Heavy metals in the vicinity of a chlor-alkali factory in the upper Negro River ecosystem, Northern Patagonia, Argentina. Science of the Total Environment 301(1-3):187-203.

Betancourt, O., Narváez, A. \& Roulet. M. 2005. Small-scale Gold Mining in the Puyango River Basin, Southern Ecuador: A Study of Environmental Impacts and Human Exposures. EcoHealth, 2(4):323-332. 
Bisinoti, M., SARgentini, E.\& JARdim. W. 2007. Seasonal Behavior of Mercury Species in Waters and Sediments from the Negro River Basin, Amazon, Brazil. Journal of the Brazilian Chemical Society 18(3):544-553.

Bloom, N., Preus, E., Katon, E. \& Hiltner, J. 2003. Selective extractions to assess the biogeochemically relevant fractionation of inorganic mercury in sediments and soils. Analytica Chimica Acta 479:233-248.

Chalén, F. 2010. Mercurio en sedimentos del litoral ecuatoriano, crucero de prospección pesquera T03/10/02D. Revistas Ciencias del Mar y Limnología 4(2):45-66.

De La Lanza, G. 1980. Materia orgánica en una laguna de la costa de Sinaloa, México (I): cuantificación total. Boletín del Instituto Oceanográfico, Sao Paulo 29(2):217-222.

Environment Canada. 1990. Guidance Document on Control of Toxicity Tes Presicion Using Reference Toxicants. Evironmental Protection Series, (3): 123-124.

Harari, R., Harari, F., Gerhardsson, L., Lundh, T., Skerfving, S., Strömberg, U. \& Broberg, K. 2012. Exposure and toxic effects of elemental mercury in gold-mining activities in Ecuador. Toxicology Letters 213(1):75-82.

Ето, K. (2000). Minamata disease. Neuropathology 20: 14-19.

IADC/CEDA. 1992. Guide 2-Conventions, Codes and Conditions, Merine Diposal and Land Diposal. International Association of Dredging Companies/Central Dredging Association.

ISO 11465. 1993. Soil quality. Determination of dry matter and water content on a mass basis Gravimetric method. International Organization for Standardization, Genève, Switzerland. 3 p.

Macfarlane, G., Koller, C. \& Blomberg, S. 2007. Accumulation and partitioning of heavy metals in mangroves: A synthesis of field-based studies. Chemosphere, 69(9):1454-1464.

Mariani, C. \& Pompêo, M. 2008. La calidad del sedimento: La contaminación por metales puede ser una amenaza para los seres vivos. Revista Ciencia Hoy 18(107):48-53.

Márquez, A., García, O., Senior, W., Martínez, G. \& González, A. 2012. Distribución de metales pesados en sedimentos superficiales del Orinoco Medio, Venezuela. Ciencia 20(1):60-73.

Monikh, F.A., Karami, O., Hosseini, M., Karami, N., Bastami, A.A. \& GHasemi, A.F. 2013. The effect of primary producers of experimental aquatic food chains on mercury and PCB153 biomagnification. Ecotoxicology and Environmental Safety 94:112-115.

Muniz, P., Danulat, E., Yannicelli, B., García-Alonso, J., Medina, G. \& Bícego, M.C. 2004. Assessment of contamination by heavy metals and petroleum hydrocarbons in sediments of Montevideo Harbour (Uruguay). Environment International 29:1019-1028.

Pawlas, N., Strömberg, U., Carlberg, B., Cerna, M., Harari, F., Harari, R., Horvat, M., Hruba, F., Koppova, K., Krskova, A., Krsnik, M., Li ,Y.F., Löfmark, L., Lundh, T., Lundström, N.G., Lyoussi, B., Markiewicz-Górka, I., Mazej, D., Osredkar, J., Pawlas, K., Rentschler, G., Spevackova, V., Spiric, Z., Sundkvist, A., Tratnik, J.S., Vadla, D., Zizi, S., Skerfving, S. \& Bergdahl. I.A. 2013.
Cadmium, mercury and lead in the blood of urban women in Croatia, the Czech Republic, Poland, Slovakia, Slovenia, Sweden, China, Ecuador and Morocco. International Journal of Occupational Medicine and Environmental Health 26(1):58-72.

Peluso, M. 2011. Evaluación de efectos biológicos y biodisponibilidad de contaminantes en sedimentos del Río de la Plata y afluentes. Tesis Doctoral. Departamento de Ciencias Biológicas. Facultad de Ciencias Exactas, Universidad Nacional de La Plata. La Plata, Argentina. 170 pp.

Rasabala, C. 2008. Inventario nacional de emisiones de mercurio y productos que contienen mercurio. Instituto de las Naciones Unidas para la Formación e Investigación (UNITAR) y el Ministerio del Ambiente. Ecuador. 221 pp.

RAVICHANDRAN, M. 2004. Interactions between mercury and dissolved organic matter- a review. Chemosphere 55(3):319331.

Rojas, L., Chang, I., Agard, J., Bekele, I. \& Hubbard, R. 2002. Heavy metals in green mussel (Perna viridis) and oysters (Crassostrea sp.) from Trinidad and Venezuela. Archives of Environmental Contamination and Toxicology 42:410415.

Convenio de Rotterdam 2004. Convenio de Rotterdam sobre el procedimiento de consentimiento fundamentado previo aplicable a ciertos plaguicidas y productos químicos peligrosos objeto de comercio internacional. Programa de las Naciones Unidas para el Medio Ambiente y Organización de las Naciones Unidas para la Agricultura y la Alimentación. Rotterdam, Holanda.

Sandoval, F., 2001. La Pequeña Minería en el Ecuador. En: International Institute for Environment and Development (IIED) and World Business Council for Sustainable Development (WBCSD) (Ed.), Mining, Minerals and Sustainable Development (MMSD).31 pp.

Schäfer, J., Blanc, G., Audry, S., Cossa, D. \& Bossy, C. 2006. Mercury in the Lot-Garonne River system (France): sources, fluxes and anthropogenic component. Applied Geochemistry 21:515-527.

Skyllberg, U., Xia, K., Bloom, P. R., Nater, E. A. \& Bleam, W. F. 2000. Binding of mercury (II) to reduced sulfur in soil organic matter along upland-peat soil transects. Journal of Environmental Quality 29(3):855-865.

Tarras-Wahlberg, N. H., Flachier, A., Lane, S. N. \& Sangfors, O. 2001. Environmental impacts and metal exposure of aquatic ecosystems in rivers contaminated by small scale gold mining: the Puyango River basin, southern Ecuador. Science of the Total Environment 278(1):239-261.

Thera, J. C. \& Rumbold, D. G. 2014. Biomagnification of mercury through a subtropical coastal food web off Southwest Florida. Environmental Toxicology and Chemistry 33(1):65-73

Vane, C., Harrison, A., Kim, V., Moss-Hayes, B. \& Long. K. 2009. Organic and metal contamination in surface mangrove sediments of South China. Marine Pollution Bulletin 50:129-166.

WHO. 1991. Inorganic Mercury. Environmental Health Criteria, 118. World Health Organization. Geneva. 